\title{
Avaliação auditiva na Síndrome da Imunodeficiência Adquirida
}

\section{Hearing evaluation in Acquired Immunodeficiency Syndrome}

\author{
Carla Gentile Matas ${ }^{1}$, Bruna de Almeida Marcon², Sara Manami Silva ${ }^{3}$, Isabela Crivellaro Gonçalves ${ }^{4}$
}

\begin{abstract}
RESUMO
Objetivo: Caracterizar as manifestações audiológicas em adultos com HIV/AIDS e comparar os resultados de indivíduos expostos e não expostos ao tratamento anti-retroviral. Métodos: Foram submetidos à avaliação audiológica 56 indivíduos com HIV/AIDS de ambos os gêneros, entre 18 e 58 anos de idade, divididos em dois grupos: GEI - composto por 24 indivíduos não expostos ao tratamento anti-retroviral; e GEII - composto por 32 indivíduos expostos ao tratamento anti-retroviral. Resultados: Verificou-se ocorrência de resultados alterados na avaliação audiológica em ambos os grupos, principalmente na audiometria de altas frequências. Em ambos os grupos a principal alteração encontrada foi a perda auditiva neurossensorial. O GEII apresentou mais resultados alterados em todos os procedimentos realizados e maior ocorrência de resultados sugestivos de alterações na orelha média, quando comparado ao GEI. Conclusão: Indivíduos com HIV/AIDS apresentam alterações auditivas periféricas, sendo estas mais acentuadas no grupo exposto ao tratamento anti-retroviral.
\end{abstract}

Descritores: HIV; Síndrome da imunodeficiência adquirida/complicações; Terapia anti-retroviral de alta atividade/efeitos adversos; Audição; Perda auditiva

\section{INTRODUÇÃO}

A Síndrome da Imunodeficiência Adquirida (AIDS) tem como agente causador um retro vírus específico, o HIV (Human Immunodeficiency Virus), que afeta o sistema imunológico, propiciando a ocorrência de diversas infecções oportunistas. Os primeiros casos de AIDS foram descritos em 1981, até a doença adquirir caráter de pandemia ${ }^{(1)}$. O Brasil é um dos países com maiores incidências da doença ${ }^{(2)}$. As infecções oportunistas, as drogas anti-retrovirais e os medicamentos po-

Trabalho realizado no Laboratório de Investigação Fonoaudiológica em Potenciais Evocados Auditivos do Curso de Fonoaudiologia do Departamento de Fisioterapia, Fonoaudiologia e Terapia Ocupacional da Faculdade de Medicina da Universidade de São Paulo - USP - São Paulo (SP), Brasil.

(1) Doutora, Professora Associada do Curso de Fonoaudiologia da Faculdade de Medicina da Universidade de São Paulo - USP - São Paulo (SP), Brasil.

(2) Especialista em Audiologia; Fonoaudióloga clínica - Caxias do Sul (RS), Brasil.

(3) Especialista em Audiologia; Fonoaudióloga colaboradora do Laboratório de Investigação Fonoaudiológica em Potenciais Evocados Auditivos do Curso de Fonaudiologia do Departamento de Fisioterapia, Fonoaudiologia e Terapia Ocupacional da Faculdade de Medicina da Universidade de São Paulo - USP - São Paulo (SP), Brasil.

(4) Pós-graduanda (Doutorado) em Ciências pelo Programa de Pós-Graduação em Ciências da Reabilitação - Área de Concentração: Comunicação Humana do Departamento de Fisioterapia, Fonoaudiologia e Terapia Ocupacional da Faculdade de Medicina da Universidade de São Paulo - USP - São Paulo (SP), Brasil.

Endereço para correspondência: Carla Gentile Matas. R. Princesa Isabel, 17/204-A, São Paulo (SP), Brasil, CEP:04601-000.E-mail: cgmatas@ usp.br Recebido em: 2/6/2009; Aceito em: 3/7/2009 tencialmente ototóxicos usados no tratamento da doença $a^{(3-8)}$, ou mesmo a ação direta do próprio HIV ${ }^{(9-13)}$ podem comprometer o sistema auditivo periférico e/ou central.

A incidência de alteração auditiva em pacientes com HIV/ AIDS varia de 20 a $40 \%{ }^{(9-11,14)}$, sendo que a perda auditiva pode ser decorrente de alterações de orelha externa, orelha média e/ ou orelha interna ${ }^{(15)}$. Com o avanço das drogas anti-retrovirais, houve o retardo da doença e melhoria da condição clínica do paciente portador do HIV devido à inibição da replicação do vírus. Autores relataram que uma tendência observada foi a presença de comprometimento auditivo em indivíduos submetidos a terapias anti-retrovirais, estando também estas alterações relacionadas à idade e o histórico de otites ${ }^{(3)}$.

$\mathrm{O}$ presente estudo teve como objetivo caracterizar as manifestações audiológicas em adultos com HIV/AIDS, comparando os resultados obtidos no grupo exposto ao tratamento anti-retroviral com aqueles obtidos no grupo não exposto ao tratamento anti-retroviral.

\section{MÉTODOS}

Este estudo foi desenvolvido no Laboratório de Investigação Fonoaudiológica em Potenciais Evocados Auditivos do Curso de Fonoaudiologia, do Departamento de Fisioterapia, Fonoaudiologia e Terapia Ocupacional da Faculdade de Medicina da Universidade de São Paulo, cujo projeto recebeu parecer favorável da Comissão de Ética para Análise de Projetos de Pesquisa - CAPPesq da Diretoria Clínica do Hospital das 
Clínicas e da Faculdade de Medicina da Universidade de São Paulo, sob protocolo número 1026/04.

Foram avaliados 56 indivíduos com HIV/ AIDS, encaminhados pela Casa da AIDS - Fundação Zerbini, com idades entre 18 e 58 anos, divididos em dois grupos: grupo estudo I (GEI), composto por 24 sujeitos não expostos ao tratamento anti-retroviral; e grupo estudo II (GEII), composto por 32 sujeitos expostos ao tratamento anti-retroviral. Os participantes assinaram inicialmente um termo de consentimento livre e esclarecido, no qual estavam descritos todos os procedimentos a serem executados.

As avaliações foram realizadas em um único dia para evitar que intercorrências como acúmulo de cerúmen, otites, disfunção tubária, perfuração de membrana timpânica, entre outras, pudessem interferir na base de informações sobre o sistema auditivo. Os procedimentos de coleta de dados incluíram: anamnese, meatoscopia, audiometria tonal convencional, audiometria de altas frequências e medidas de imitância acústica. Os equipamentos utilizados para realização destes procedimentos foram: otoscópio da marca Heine®, analisador de orelha média GSI-33 marca Grason Stadler® e audiômetro GSI-61 marca Grason Stadler®. Os parâmetros analisados para cada procedimento encontram-se a seguir:

- Audiometria tonal convencional: limiares de audibilidade nas frequências de $250 \mathrm{~Hz}$ a $8000 \mathrm{~Hz}$ por via aérea e 500 $\mathrm{Hz}$ a $4000 \mathrm{~Hz}$ por via óssea, quando os limiares de via aérea encontravam-se rebaixados;

- Audiometria de altas frequências: limiares de audibilidade nas frequências de 9, 10, 11.2, 12.4, 14, 16, 18 e 20 kHz;

- Medidas de imitância acústica: curva timpanométrica e limiares dos reflexos acústicos contralaterais e ipsilaterais nas frequências de 500, 1000, 2000 e $4000 \mathrm{~Hz}$.

A audiometria de altas frequências não foi realizada em cinco indivíduos do GEI e dez do GEII, por indisponibilidade em realizar todos os exames propostos na pesquisa.

Os resultados das avaliações audiológicas foram classificados como normais ou alterados, de acordo com os padrões referidos na literatura para as medidas de imitância acústica ${ }^{(16)}$; audiometria tonal ${ }^{(17)}$; e audiometria de altas frequências ${ }^{(18)}$.O indivíduo foi considerado alterado quando pelo menos uma das orelhas apresentava alteração.

Posteriormente, foram descritos os tipos de alterações encontradas na avaliação audiológica básica, levando-se em consideração os resultados obtidos na audiometria tonal convencional e medidas de imitância acústica, sendo classificados como perda auditiva do tipo condutiva, neurossensorial ou $\operatorname{mista}^{(17)}$. A perda auditiva foi considerada neurossensorial quando os limiares de audibilidade obtidos por via aérea e via óssea encontravam-se rebaixados, com ausência de gap entre os mesmos. A perda auditiva foi considerada condutiva quando os limiares de audibilidade por via aérea encontravam-se rebaixados e os limiares por via óssea encontravam-se dentro dos padrões de normalidade. A perda auditiva foi considerada mista quando ambos os limiares, por via aérea e óssea, encontravam-se rebaixados, com presença de gap entre eles. Nos casos em que o mesmo indivíduo apresentou mais de um tipo de alteração (ou seja, um tipo de perda auditiva em cada orelha), a alteração foi classificada da seguinte maneira: perda auditiva neurossensorial associada à perda auditiva condutiva; perda auditiva neurossensorial associada à perda auditiva mista ou perda auditiva condutiva associada à perda auditiva mista.

Os dados foram submetidos à análise estatística por meio dos testes t de Student e Qui-quadrado. O nível de significância adotado foi de $5 \%$.

\section{RESULTADOS}

Verificou-se prevalência de perda auditiva em 41,1\% do total de indivíduos com HIV/AIDS avaliados. Entre os indivíduos que apresentaram perda auditiva, $39,1 \%$ pertenciam ao grupo não exposto ao tratamento anti-retroviral e 60,9\% pertenciam ao grupo exposto ao tratamento anti-retroviral; $43,5 \%$ apresentaram comprometimento unilateral e 56,5\% bilateral.

Levando-se em consideração os resultados da audiometria tonal convencional e medidas de imitância acústica, observouse maior ocorrência de resultados alterados no GEII $(43,75 \%)$ quando comparado com o GEI $(37,5 \%)$ sem diferença estatisticamente significante entre os grupos $(p=0,638)$ (Tabela 1$)$. Além disso, verificou-se que no GEI houve predominância de perda auditiva nas frequências altas $(66,7 \%)$ e no GEII, perda auditiva do tipo neurossensorial (42,8\%) (Tabela 2).

$\mathrm{Na}$ audiometria de altas frequências, verificou-se frequente ocorrência de resultados alterados em ambos os grupos (Tabela 3), sem diferença estatisticamente significante quando se comparou os grupos $(\mathrm{p}=0,226)$. Note-se que o grupo GEII

Tabela 1. Ocorrência de resultados normais e alterados na avaliação audiológica convencional (audiometria tonal e medidas de imitância acústica

\begin{tabular}{lcccc}
\hline Resultado & \multicolumn{2}{c}{ Grupo I } & \multicolumn{2}{c}{ Grupo II } \\
\cline { 2 - 5 } & $\mathrm{N}$ & $\%$ & $\mathrm{~N}$ & $\%$ \\
\hline Normal & 15 & 62,5 & 18 & 56,25 \\
Alterado & 9 & 37,5 & 14 & 43,75 \\
\hline Valor de $\mathrm{p}$ & \multicolumn{3}{c}{$0,638\left(\mathrm{X}^{2}=0,221 ;\right.$ n.g.l. $\left.=1\right)$} \\
\hline
\end{tabular}

Legenda: $\mathrm{N}=$ número de indivíduos

Tabela 2. Tipos de alterações observadas na avaliação audiológica convencional (audiometria tonal e medidas de imitância acústica)

\begin{tabular}{lccccc}
\hline Grupo & $\begin{array}{c}\text { Perda auditiva NS } \\
\text { (unilateral ou bilateral) }\end{array}$ & $\begin{array}{c}\text { Perda auditiva condutiva } \\
\text { (unilateral ou bilateral) }\end{array}$ & $\begin{array}{c}\text { NS + mista } \\
\text { NS + condutiva } \\
\text { Mista + condutiva }\end{array}$ \\
\cline { 2 - 6 } & $\mathrm{N}$ & $\%$ & $\mathrm{~N}$ & $\mathrm{~N}$ & $\mathrm{~N}$ \\
\hline Grupo I & 2 & 22,2 & 1 & 11,1 & 0 \\
Grupo II & 6 & 42,8 & 4 & 28,6 & 6 \\
\hline
\end{tabular}

Legenda: $\mathrm{NS}=$ neurossensorial; N= número de indivíduos 
apresentou maior ocorrência de resultados alterados $(95,5 \%)$ que o GE1 $(84,2 \%)$.

Tabela 3. Ocorrência de resultados normais e alterados na audiometria de altas frequências

\begin{tabular}{lcccc}
\hline Resultado & \multicolumn{2}{c}{ Grupo I } & \multicolumn{2}{c}{ Grupo II } \\
\cline { 2 - 5 } & $\mathrm{N}$ & $\%$ & $\mathrm{~N}$ & $\%$ \\
\hline Normal & 3 & 15,8 & 1 & 4,5 \\
Alterado & 16 & 84,2 & 21 & 95,5 \\
\hline Valor de $\mathrm{p}$ & \multicolumn{3}{c}{$0,226\left(\mathrm{X}^{2}=1,464 ;\right.$ n.g.l. $\left.=1\right)$} \\
\hline
\end{tabular}

Legenda: $\mathrm{N}$ = número de indivíduos

Quando considerada a média dos limiares tonais obtidos na audiometria de altas frequências, verificou-se diferença estatisticamente significante entre os grupos para a frequência de $18 \mathrm{kHz}$, e tendência à diferença estatisticamente significante para as frequências de 16 e $20 \mathrm{kHz}$ (Tabela 4).

Tabela 4. Comparação entre os grupos I e II com relação aos limiares de audibilidade

\begin{tabular}{lccccc}
\hline Altas frequências & Média & DP & N & Valor de p \\
\hline $9 \mathrm{kHz}$ & Grupo I & 35,53 & 33,04 & 19 & 0,705 \\
& Grupo II & 38,41 & 35,22 & 22 & \\
$10 \mathrm{kHz}$ & Grupo I & 32,76 & 31,38 & 19 & 0,510 \\
& Grupo II & 37,61 & 34,48 & 22 & \\
$11,2 \mathrm{kHz}$ & Grupo I & 35,39 & 31,35 & 19 & 0,265 \\
& Grupo II & 43,52 & 33,85 & 22 & \\
$12,5 \mathrm{kHz}$ & Grupo I & 41,84 & 31,70 & 19 & 0,539 \\
& Grupo II & 46,36 & 34,29 & 22 & \\
$14 \mathrm{kHz}$ & Grupo I & 45,66 & 28,55 & 19 & 0,236 \\
& Grupo II & 53,06 & 27,52 & 22 & \\
$16 \mathrm{kHz}$ & Grupo I & 41,84 & 22,31 & 19 & $0,060 \#$ \\
& Grupo II & 50,11 & 16,83 & 22 & \\
$18 \mathrm{kHz}$ & Grupo I & 25,26 & 13,75 & 19 & $0,009^{*}$ \\
& Grupo II & 32,16 & 9,61 & 22 & \\
$20 \mathrm{kHz}$ & Grupo I & 11,45 & 8,54 & 19 & $0,080 \#$ \\
& Grupo II & 14,55 & 7,30 & 22 & \\
\hline \multirow{2}{*}{$\mathrm{Val}$. } & & &
\end{tabular}

* Valores estatisticamente significantes

\# Valores com tendência à diferença estatisticamente significante

Legenda: $\mathrm{N}=$ número de indivíduos; $\mathrm{DP}=$ desvio-padrão

\section{DISCUSSÃO}

Entre as manifestações auditivas e vestibulares mais frequentes em portadores do HIV estão: perda auditiva (62\%), otorréia (31\%), vertigens (15\%) e zumbido (15\%), sendo que as perdas auditivas nesta população podem ser condutivas ou neurossensoriais ${ }^{(19)}$. Os resultados da presente pesquisa indicaram, assim como outros estudos, que a deficiência auditiva em indivíduos com HIV/AIDS pode ser variável quando considerados tipo, grau e configuração da perda auditiva; no entanto, em geral, esta se inicia nas frequências mais altas e evolui acometendo as demais frequências ${ }^{(20)}$.

Observam-se, na literatura especializada, resultados bastan- te variados com relação à prevalência de perda auditiva em indivíduos com HIV/AIDS. No presente estudo, verificou-se uma prevalência de perda auditiva em 41,1\% dos indivíduos com maior frequência de comprometimento bilateral. Observou-se maior prevalência de alteração auditiva no grupo de indivíduos expostos ao tratamento anti-retroviral $(43,75 \%)$ quando comparado ao grupo de indivíduos não expostos $(37,5 \%)$ (Tabela 1). Nos indivíduos soropositivos para o HIV, em geral as perdas auditivas neurossensoriais são progressivas e bilaterais, podendo ser decorrentes de doenças oportunistas ou de medicações ototóxicas $^{(3)}$. Em dois estudos a alteração auditiva afetou até $33 \%$ destes pacientes ${ }^{(9,10)}$. Em outro, verificou-se menor prevalência de alterações otológicas em indivíduos infectados pelo HIV, sendo esta de $6,8 \%$ no grupo tratado com terapia anti-retroviral e 3,2\% do grupo não tratado, com ausência de diferença estatisticamente significante entre os grupos ${ }^{(21)}$. Em pesquisa realizada com indivíduos infectados pelo HIV, não foram encontradas alterações na audição destes, avaliados 16 e 32 semanas após o início da terapia anti-retroviral ${ }^{(22)}$.

Entre todos os indivíduos com HIV/AIDS avaliados, 28,6\% apresentaram algum tipo de comprometimento neurossensorial na audiometria tonal convencional (com prevalência de 33,3\% no grupo não exposto ao tratamento anti-retroviral e $25 \%$ no grupo exposto ao tratamento anti-retroviral). Considerando-se a classificação adotada no presente estudo, no grupo I a manifestação mais frequente neste tipo de comprometimento foi a perda auditiva nas frequências altas, que representou $66,7 \%$ das alterações encontradas. No grupo II, o tipo de alteração de maior ocorrência foi a perda auditiva neurossensorial, representando $42,8 \%$ das alterações (Tabela 2).

O comprometimento misto foi apresentado por $5,4 \%$ dos indivíduos com HIV/AIDS avaliados (todos pertencentes ao grupo exposto ao tratamento anti-retroviral), e esteve associado às perdas auditivas neurossensorial e/ou condutiva (Tabela 2).

Em relação às alterações da orelha interna, pesquisadores referem que o envolvimento neurossensorial da audição em pacientes infectados pelo HIV varia de $20,9 \%$ a $49 \%$, poucos constatam perda auditiva neurossensorial súbita ${ }^{(14)}$. Autores atribuíram como mecanismo para perda auditiva associada à terapia anti-retroviral, a lesão do DNA mitocondrial ${ }^{(3)}$.

Os resultados da audiometria de altas frequências indicaram que $90,2 \%$ de todos os indivíduos avaliados apresentaram alteração (com prevalência de $84,2 \%$ no grupo não exposto ao tratamento anti-retroviral e $95,5 \%$ no grupo exposto ao tratamento anti-retroviral - Tabela 3), demonstrando que a perda auditiva em indivíduos com HIV/AIDS tem como característica o rebaixamento mais acentuado nas frequências altas, corroborando os dados descritos na literatura ${ }^{(3,10,13)}$. Em estudo realizado com 30 indivíduos soropositivos, foram obtidos resultados normais na audiometria convencional; no entanto, a audiometria de altas frequências mostrou alterações neurossensoriais em $23 \%$ dos pacientes ${ }^{(13)}$. Apesar da dificuldade em se estabelecer critérios de normalidade fidedignos para a audiometria de altas frequências, visto a grande variabilidade entre sujeitos, este teste tem se mostrado útil na avaliação da progressão do acometimento auditivo decorrente de ototoxicidade, em especial nos indivíduos submetidos a tratamento quimioterápico, uma vez que a alteração inicia-se nas frequências 
altas e progride para as mais baixas, de acordo com a dosagem cumulativa da droga ${ }^{(23)}$. Portanto, considerando-se a elevada prevalência de resultados alterados na audiometria de altas frequências, principalmente naqueles indivíduos expostos ao tratamento anti-retroviral, o uso da mesma no monitoramento da audição de indivíduos com HIV/AIDS deve ser considerado. A identificação precoce de alterações auditivas nesta população possibilitaria a elaboração de estratégias terapêuticas mais adequadas, principalmente com relação às orientações fornecidas ao paciente e/ou sua família.

Um outro achado que deve ser levado em consideração está relacionado à presença de diferença estatisticamente entre os grupos para a média dos limiares obtidos na frequência de 18 $\mathrm{kHz}$ e uma tendência à diferença estatisticamente significante para as frequências de 16 e $20 \mathrm{kHz}$. Tais resultados reforçam a idéia de que a ocorrência de perda auditiva nesta população está relacionada ao grau de acometimento do vírus ${ }^{(9,10)}$, bem como à ototoxicidade das drogas utilizadas na terapia anti-retroviral.

Observou-se algum tipo de comprometimento condutivo em 33,3\% dos indivíduos que apresentaram alterações auditivas (com prevalência de 4,2\% no grupo não exposto ao tratamento anti-retroviral e $21,9 \%$ no grupo exposto ao tratamento anti-retroviral). Tal comprometimento ocorreu de maneira isolada ou concomitantemente ao comprometimento neurossensorial (perdas auditivas mistas ou perdas auditivas condutivas associadas às perdas neurossensoriais ou mistas). Quando considerada sua ocorrência isolada, representou 11,1\% das alterações encontradas no grupo I e $28,6 \%$ no grupo II (Tabela 2). Tais achados corroboram aqueles descritos na literatura, que referem como manifestações frequentes nestes indivíduos as otites externa e média, mastoidite, perfuração da membrana timpânica e colesteatoma ${ }^{(24)}$. Em pesquisa realizada, verificou-se que $8 \%$ dos indivíduos avaliados apresentaram evidências de otite média crônica, geralmente com efusão ${ }^{(20)}$.

Considerando-se o quadro clínico dos indivíduos expostos ao tratamento anti-retroviral, tais resultados podem estar re- lacionados ao fato de se encontrarem imunodeprimidos e, em decorrência desta condição, apresentarem com mais frequência alterações de orelha média. Em contrapartida, outros pesquisadores avaliaram o impacto das novas drogas anti-retrovirais na prevalência de otite média crônica em crianças infectadas pelo HIV, e verificaram que o tratamento esteve associado à menor prevalência desta doença em crianças menores de seis anos, apontando a eficácia do tratamento anti-retroviral no combate a infecções, como otites ${ }^{(25)}$.

Apesar de serem frequentes tais alterações de orelha média em indivíduos com HIV/AIDS, é importante ressaltar que, as manifestações decorrentes do HIV propriamente dito são raras, sendo mais encontradas as doenças oportunistas, como a otite externa, otite média aguda, otite média recorrente, otite média com efusão, otite média crônica supurativa colesteatomatosa e herpes zoster ótico ${ }^{(26)}$.

Considerando-se a variabilidade de achados obtidos na avaliação audiológica em indivíduos com HIV/AIDS, torna-se necessária a realização de pesquisas que investiguem o modo pelo qual a infecção pelo vírus, as doenças oportunistas e uso de medicamentos ototóxicos atuam no sistema auditivo periférico nesta população. Além disso, visto o crescente desenvolvimento de drogas que permitem o retardo da doença e, consequentemente, o aumento da expectativa de vida destes indivíduos, deve-se salientar que a identificação precoce de alterações auditivas pode contribuir para um melhor prognóstico, assim como no processo de reabilitação destes pacientes.

\section{CONCLUSÃO}

Os indivíduos com HIV apresentaram alterações sugestivas de comprometimento da via auditiva periférica e dos limiares de audibilidade em alta frequência. Esses resultados sugerem que a audiometria de altas frequências é um dos testes mais sensíveis para identificação precoce de alterações auditivas nesta população.

\begin{abstract}
Purpose: To characterize audiological manifestations in adults with HIV/AIDS and to compare the results of subjects exposed and not exposed to antiretroviral treatment. Methods: Fifty six individuals with HIV/AIDS of both genders, with ages between 18 and 58 years, were submitted to audiological evaluation. Subjects were divided into two groups: GEI - composed of 24 individuals not exposed to antiretroviral treatment; and GEII - composed of 32 individuals exposed to antiretroviral treatment. Results: It was verified occurrence of altered results on audiological evaluation in both groups, mainly in the high frequencies audiometry. In both groups, the main alteration found was sensorineural hearing loss. The GEII showed more altered results in all procedures performed, and higher occurrence of findings suggestive of alterations in middle ear, when compared to GEI. Conclusion: Individuals with HIV/AIDS in this study had peripheral hearing impairment, more pronounced in the group exposed to antiretroviral treatment.
\end{abstract}

Keywords: HIV; Acquired immunodeficiency syndrome/complications; Antiretroviral therapy, highly active/adverse effects; Hearing; Hearing loss

\title{
REFERÊNCIAS
}

1. World Health Organization - Global HIV/AIDS e STD Surveillance [Internet] 1998. [cited 2010 May 29]. Available from URL: http://www. who.int/emc-hiv/global_report/slides/
2. Brasil. Ministério da Saúde. Coordenação Nacional DST/AIDS. Bol Epidemiol - AIDS e DST. 2007; 4(1 jan/jun). 
3. Marra CM, Wechkin HA, Longstreth WT Jr, Rees TS, Syapin CL, Gates GA. Hearing loss and antiretroviral therapy in patients infected with HIV-1. Arch Neurol. 1997;54(4):407-10.

4. Christensen LA, Morehouse CR, Powell TW, Alchediak T, Silio M. Antiviral therapy in a child with pediatric human immunodeficiency virus (HIV): case study of audiologic findings. J Am AcadAudiol. 1998;9(4):292-8.

5. Vogeser M, Colebunders R, Depraetere K, Van Wanzeele P, Van Gehuchten S. Deafness caused by didanosine. Eur J ClinMicrobiol Infect Dis. 1998;17(3):214-5.

6. Simdon J, Watters D, Bartlett S, Connick E. Ototoxicity associated with use of nucleoside analog reverse transcriptase inhibitors: a report of 3 possible cases and review of the literature. Clin Infect Dis. 2001;32(11):1623-7. Comment in: Clin Infect Dis. 2001;33(12):2100-2. Clin Infect Dis. 2002;34(3):418-9.

7. Williams B. Ototoxicity may be associated with protease inhibitor therapy. Clin Infect Dis. 2001;33(12):2100-2.

8. Rey D, L'Héritier A, Lang JM. Severe ototoxicity in a health care worker who received postexposure prophylaxis with stavudine, lamivudine, and nevirapine after occupational exposure to HIV. Clin Infect Dis. 2002;34(3):418-9. Comment in: Clin Infect Dis. 2001;32(11):1623-7.

9. Chandrasekhar SS, Connelly PE, Brahmbhatt SS, Shah CS, Kloser PC, Baredes S. Otologic and audiologic evaluation of human immunodeficiency virus-infected patients. Am J Otolaryngol. 2000;21(1):1-9.

10. Mata Castro N, YebraBango M, Tutor de Ureta P, Villarreal GarcíaLomas M, GarcíaLópez F. [Hearing loss and human immunodeficiency virus infection. Study of 30 patients]. Rev Clin Esp. 2000;200(5):271-4. Spanish.

11. Khoza K, Ross E. Auditory function in a group of adults infected with HIV/AIDS in Gauteng, South Africa. S Afr J CommunDisord. 2002;49:17-27.

12. Birchall MA, Wight RG, French PD, Cockbain Z, Smith SJ. Auditory function in patients infected with the human immunodeficiency virus. ClinOtolaryngol Allied Sci. 1992;17(2):117-21.

13. Domènech J, Fusté J, Traserra J. [Equilibrium and auditory disorders in patients affected by HIV-1]. Rev Neurol. 1996;24(136):1623-6. Spanish.
14. Madriz JJ, Herrera G. Human immunodeficiency virus and acquired immune deficiency syndrome AIDS - related hearing disorders. J Am AcadAudiol. 1995;6(5):358-64. Review.

15. Matkin ND, Diefendorf AO, Erenberg A. Children: HIV/AIDS and hearing loss. Semin Hear. 1998;19(2):143-53.

16. Jerger J. Clinical experience with impedance audiometry. Arch Otolaryngol. 1970;92(4):311-24.

17. Santos TMM, Russo ICP. Logoaudiometria. In: Santos TMM, Russo ICP. A prática da audiologia clínica. 3a ed. São Paulo: Cortez Editora; 1991. p. 73-88.

18. Carvallo RMM. Audição em altas frequências: repercussões no reconhecimento de fala no ruído e nas emissões otoacústicas [tese]. São Paulo: Faculdade de Medicina da Universidade de São Paulo; 2002.

19. Salzer TA. Neurotologic manifestations of HIV infection. Grand Rounds Arch. 1994;24:1-5.

20. Soucek S, Michaels L. The ear in the acquired immunodeficiency syndrome: II. Clinical and audiologic investigation. Am J Otol. 1996;17(1):35-9.

21. Vieira ABC, Greco DB, Teófilo MMM, Gonçalves DU. Manifestações otoneurológicas associadas à terapia anti-retroviral. Rev Soc Bras Med Trop.2008;41(1):65-9.

22. Schouten JT, Lockhart DW, Rees TS, Collier AC, Marra CM. A prospective study of hearing changes after beginning zidovudine or didanosine in HIV-1 treatment-naïve people. BMC Infect Dis. 2006;6:28.

23. Garcia AP, Iório MCM, Petrilli AS. Monitoramento da audição de pacientes expostos à cisplatina. Rev Bras Otorrinolaringol. 2003;69(2):215-21.

24. Gold S, Tami TA. Otolaryngological manifestations of HIV/AIDS. Semin Hear.1998;19(2):165-75.

25. Weber R, Pinheiro Neto CD, Miziara ID, Araújo Filho BC. Impacto da Haart na prevalência de otite média crônica em crianças brasileiras infectadas pelo HIV. Rev Bras Otorrinolaringol. 2006;72(4):509-14.

26. Morris MS, Prasad S. Otologic disease in the acquired immunodeficiency syndrome. Ear Nose Throat J. 1990;69(7):451-3. Review. 